\title{
Défibrillation à l'aide de défibrillateurs auto- matiques et semi-automatiques externes (AED)
}

\author{
Extrait des directives et recommandations pour la formation et la pratique. Texte intégral à l'adresse suivante: \\ www.resuscitation.ch
}

Erwin Oechslin, Zurich (président); Lion Bernoulli, Zurich; Urs Klemmer, Kloten; Roberto Malacrida, Lugano; Joseph Osterwalder, St-Gall; Martin von Planta, Bâle; Philippe Reigner, Lausanne; Gian Antonio Romano, Verscio; Hugo Saner, Olten; Wolfgang Ummenhofer, Bâle; Markus Wietlisbach, Sursee

Deutsch erschienen

in SÄZ Nr. 39/2001

\section{Introduction}

La fibrillation ventriculaire est la cause la plus fréquente (jusqu'à 80\%) de l'arrêt circulatoire soudain qui peut apparaître sans symptômes ou dans l'heure qui suit le début des symptômes. Alors que les chances de succès d'une réanimation dans le cas d'une asystolie ou d'une dissociation électro-mécanique sont minimes, la défibrillation immédiate d'une fibrillation ventriculaire - seule méthode de traitement - offre une chance réelle de survie.

L'élément déterminant d'une défibrillation réussie est l'intervalle entre la survenue de la fibrillation ventriculaire et la défibrillation. Chaque minute de retard diminue les chances de succès d'une défibrillation de 7 à 10\% (fig. 1).

Hormis l'appel immédiat du médecin d'urgence par le $n^{\circ} 144$ et la pratique correcte de la réanimation cardiopulmonaire de base (Basic Life Support, [BLS]), le recours au défibrillateur et à la réanimation cardiovasculaire avancée (Advanced Cardiovascular Life Support [ACLS]) sont des éléments décisifs de la réanimation. La pratique de la défibrillation par du personnel paramédical ou des profanes permet de raccourcir considérablement le délai entre la survenue de la fibrillation ventriculaire et la première défibrillation.

Les «Guidelines 2000 for Cardiopulmonary Resuscitation and Emergency Cardiovascular Care» sont des recommandations reconnues à l'échelle internationale $[1,2]$. Le BLS et la défibrillation précoce y occupent une place importante. Grâce à leur emploi simple et sûr et à une interprétation correcte du rythme cardiaque et de l'ordre de défibrillation, les défibrillateurs automatiques (automated external defibrillator [AED]) seront accessibles à un large éventail d'utilisateurs non-médecins [3]. Ce concept et la vulgarisation de la défibrillation précoce à l'aide des AED doit répondre à des exigences de qualité et de sécurité dans la formation des utilisateurs indispensables à un usage approprié de cet équipement. Ces directives et recommandations, basées sur celles de l'American Heart Association, définissent la formation à la défibrillation avec des défibrillateurs automatiques et semi-automatiques en vue de leur utilisation. Ce document ne concerne pas la formation et l'usage de la défibrillation manuelle.

\section{Aspects légaux}

\subsection{Personnel paramédical}

La défibrillation par le personnel paramédical se fonde en Suisse de manière incontestable, sur le plan juridique, sur le principe de la délégation implicite de compétence. La légitimité de cette délégation repose sur la sélection adéquate des candidats à former, une instruction appropriée et un contrôle effectif [4]. Dans ce contexte, les dispositions générales relatives à la supervision médicale, à la coordination et au contrôle sont très importantes [5]. La personne à qui la tâche de défibrillation a été déléguée répond de sa pratique correcte, alors que le médecin est dans tous les cas responsable de l'acte délégué.

\subsection{Profanes}

Les profanes ne tombent pas juridiquement sous le principe de la délégation de compétence. Le droit des profanes à pratiquer la défibrillation à l'aide d'AED et la protection des profanes, des fabricants d'appareils et des médecins qui s'occupent de programmes AED contre des plaintes civiles et des demandes en dommages-intérêts nécessitent une réflexion juridique approfondie.

\subsubsection{Législation fédérale}

Droit pénal: Selon l'art. 128 du Code pénal, chacun a l'obligation de porter secours à une personne en danger de mort imminente. Se soustraire à cette obligation ou empêcher une tierce personne de porter secours est pénalement pu- 
Figure 1

Succès de la réanimation (en \%) par rapport au délai d'application.

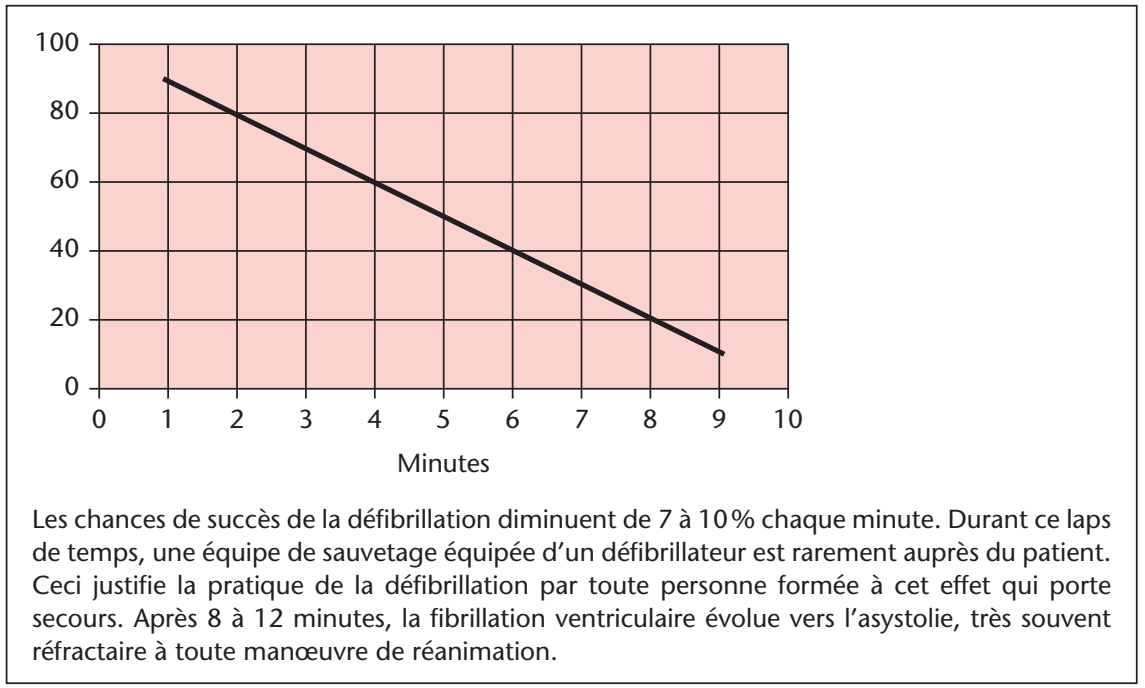

nissable. Le devoir de porter secours dépend des circonstances. Sur le plan médical, ce devoir existe lorsque l'on se soumet à une formation en BLS ou à la défibrillation à l'aide d'AED. On peut émettre l'hypothèse qu'une personne formée à l'usage du défibrillateur, qui ne l'utiliserait pas en urgence, serait punissable. L'omission de porter secours n'est cependant punissable que si elle est intentionnelle. Elle ne l'est pas dans les cas de négligence. La responsabilité juridique des fabricants d'appareils (responsabilité civile du produit) et des médecins superviseurs n'est pas encore réglée.

Droit civil: La question de la responsabilité, dans le cas d'un dommage causé à un patient ou à une tierce personne à la suite du recours à un défibrillateur, est également d'une grande importance. Une protection générale des secouristes contre les demandes en dommages-intérêts, comme dans plusieurs Etats américains (good samaritan law), n'existe pas en Suisse. Les plaintes civiles et les demandes en dommages-intérêts sont donc possibles.

\subsubsection{Législations cantonales}

Même dans le cadre de la nouvelle constitution fédérale, les lois touchant le secteur sanitaire demeurent de la compétence des cantons, à l'exception de la LAMal et des assurances sociales. Par conséquent, la prise en charge de tout problème de santé reste soumise à un accord des autorités cantonales, y compris pour la pratique de la réanimation cardiopulmonaire (BLS) et de la défibrillation. La question se pose dès lors d'un accord obligatoire général pour l'utilisation d'un défibrillateur ou d'un accord uniquement lors d'une utilisation professionnelle ou lucrative, ainsi que des personnes auxquelles peut être délivrée cette autorisation. La réponse dépend de chaque législation cantonale. Dans le canton de St-Gall, p. ex., il n'y a que les activités de prise en charge des problèmes de santé dans le cadre d'une activité lucrative qui nécessitent une autorisation. Ainsi, il n'existe pas de disposition légale relative aux activités sanitaires sans but lucratif.

\section{Conclusions}

- La législation fédérale permet la pratique de la défibrillation à l'aide d'AED par les profanes.

- La législation fédérale ne permet pas de punir pour négligence le profane qui pratique la défibrillation lorsqu'elle relève du devoir de porter secours à une personne en danger. Il est en revanche possible de recourir, sur le plan de la responsabilité civile, contre les utilisateurs, les fabricants d'appareils et les médecins superviseurs qui doivent par conséquent être assurés de façon appropriée.

- Avant de lancer un programme AED, les autorités sanitaires cantonales (le département de la santé publique) doivent définir le cadre général dans lequel ce programme s'inscrit. Dans ce contexte, une demande d'accord de la commission d'éthique compétente est importante et utile.

- La situation sur le plan du droit civil et de la responsabilité des fabricants d'appareils (responsabilité du fait des produits) n'est pas résolue pour le moment.

- Il s'agira à l'avenir de mettre sur pied une législation pénale et civile unifiée, apte à régler les compétences et la responsabilité des médecins superviseurs, du personnel paramédical, des profanes et des fabricants d'appareils lors des manœuvres de réanimation.

\subsection{Actes médicaux délégués dans le cadre de l'activité professionnelle}

L'autorisation de pratiquer la défibrillation pour le personnel paramédical dans le cadre d'une activité professionnelle (formation dans le domaine sanitaire ou médical), ainsi que pour les secouristes dans le cadre de tâches sociales doit être délivrée par un médecin responsable. Une autorisation médicale permet ainsi à celui qui la possède de pratiquer une défibrillation par AED dans les situations d'urgence.

Les devoirs du médecin responsable comprennent: 
- la formation et la formation continue du personnel;

- l'analyse des défibrillations effectuées;

- le contrôle qualité.

\section{Types de défibrillateurs}

Les défibrillateurs automatiques externes (AED) sont des défibrillateurs externes équipés d'un module d'analyse du rythme cardiaque. Un signal acoustique ou visuel indique à l'utilisateur les manœuvres à faire. La désignation AED s'applique aux défibrillateurs automatiques et semi-automatiques:

- Les défibrillateurs automatiques analysent le rythme cardiaque et procèdent à la défibrillation de manière autonome: après l'enclenchement de l'appareil et la pose des électrodes, le fonctionnement est automatique. En cas de fibrillation ventriculaire ou de tachycardie ventriculaire, les condensateurs se chargent et l'appareil délivre un choc électrique à une énergie prédéfinie (défibrillation).

- Les défibrillateurs semi-automatiques apportent une aide à la décision en ce sens qu'ils exigent certaines manipulations ultérieures telles que l'activation de la fonction «analyse» pour l'analyse rythmique et celle de la fonction «choc» ou «décharge» pour la défibrillation effective. Après l'analyse du rythme, ils donnent une recommandation en vue de la défibrillation qui doit cependant être déclenchée par l'utilisateur.

Les défibrillateurs manuels ne fournissent aucune indication sur la procédure de décision et ne sont pas équipés d'un module d'analyse du rythme. L'interprétation du rythme, ainsi que l'indication et l'exécution de la défibrillation doivent être faites par l'utilisateur.

\section{Exigences relatives aux défibrillateurs automatiques et semi-automatiques}

Les AED doivent répondre aux exigences spécifiques suivantes:

- diagnostic correct des arythmies potentiellement létales, y compris dans des conditions difficiles;

- efficacité de l'application du choc électrique;

- suppression des abus et des erreurs de maniement grâce à la simplicité d'emploi;

- risque minime de lésion, pour le patient comme pour l'utilisateur;
- auto-contrôle avec indication de l'état de fonctionnement;

- stockage des données;

- maniabilité, facilité d'utilisation, dimensions réduites et faible poids;

- maintenance simple et prix modéré.

Une grande attention doit être portée à la sécurité. Les AED certifiés par la Federal Drug Administration (FDA) garantissent efficacité et sécurité. Des différences existent cependant pour les autres critères.

\section{Emplacement des défibrillateurs automatiques externes (AED)}

Le personnel de secours spécialement formé utilise de plus en plus les AED. Les ambulances de secours et de transfert, ainsi que les véhicules de transport de patients doivent en être équipés, s'ils ne le sont pas déjà par des défibrillateurs manuels auxquels du personnel qualifié a recours.

La présence d'AED à certains endroits stratégiques est non seulement justifiée, mais recommandée, les chances de succès d'une réanimation lors de fibrillation ventriculaire étant considérablement réduites au bout de $10 \mathrm{~min}$. Les AED des lieux publics doivent être reliés à un système d'urgence sous la responsabilité d'un médecin et être annoncés à la centrale d'alarme du n 144.

Les directives pour l'établissement d'un programme AED et pour l'emplacement des AED se fondent sur le risque et la probabilité d'un arrêt circulatoire et sur le délai d'intervention.

\subsection{Situations à risque}

- Cabinets médicaux, hôpitaux (divisions hospitalières);

- ambulances de sauvetage et de transfert, véhicules de police et de pompiers;

- logements des patients de risque;

- places de sport, centres de remise en forme, piscines, bains publics;

- zones industrielles (p.ex. centrales électriques).

4.2 Lieux publics avec grande concentration de personnes et probabilité d'un arrêt cardiaque par 1000 personnes/an

- Gares, aéroports, salles de concert, théâtres;

- zones industrielles (fabriques), complexes administratifs, centres commerciaux, stades de sport. 


\subsection{Délais d'intervention}

- Intervalle de plus de 5 min. entre l'alarme et la disponibilité d'un défibrillateur manuel (conventionnel) en ayant recours à un système conventionnel d'alarme, p.ex. dans le cas des moyens de transport (avions, trains, bateaux).

- Recours aux défibrillateurs AED par du personnel médical ou paramédical ou du personnel de secours spécialement formé, s'ils sont intégrés dans un système d'alarme, pratiquent les méthodes de BLS et la défibrillation dans les 5 min. suivant l'alarme.

\section{Formation dans le domaine des AED (cours de base)}

La formation dans le domaine de la défibrillation précoce par AED de groupes définis et l'accès aux AED (public access defibrillation) permettent d'améliorer le taux de survie après arrêt circulatoire sur fibrillation ventriculaire ou tachycardie ventriculaire sans pouls. Mais les AED ne trouvent leur utilité thérapeutique que lors d'arrêt circulatoire par fibrillation ventriculaire ou tachycardie ventriculaire sans pouls. Dans toutes les autres situations, les AED en cas d'arrêt circulatoire sont inutiles, sauf en cas de recours éventuel à un moniteur, ce qui souligne la nécessité d'une formation préliminaire et approfondie en BLS. Cette formation ne doit pas uniquement être centrée sur un programme AED, mais doit aussi inclure les situations de fibrillation ventriculaire et tachycardie ventriculaire réfractaires après défibrillation par AED, ainsi que les autres arythmies accessibles à une réanimation.

\subsection{Public-cible}

Chacun peut être formé à la défibrillation précoce par AED, y compris les jeunes à partir de 10 ans.

\subsection{Objectif des cours}

L'objectif des cours AED est d'améliorer le taux de survie des patients victimes d'un arrêt circulatoire en raccourcissant le délai entre l'arrêt circulatoire et le recours au BLS et à la défibrillation (amélioration de la chaîne de survie).

\subsection{Conditions de participation au cours de base AED}

Pour être admis à un cours de base AED, le candidat doit être porteur d'un certificat valable attestant la participation à un cours de base ou de répétition BLS. Le dernier cours de base BLS ne doit pas remonter à plus de 2 ans.

\subsection{Axes prioritaires des cours de base AED} Le programme de formation comprend un enseignement théorique, mais surtout des exercices pratiques sur mannequins, avec AED. La maîtrise du BLS et l'application correcte des défibrillateurs forment les axes prioritaires des cours.

La durée optimale du cours est de 4 heures, selon les recommandations de l'annexe 1. L'instructeur du cours peut adapter son programme en fonction des connaissances des participants et des besoins locaux. Les exigences minimales doivent être remplies (ABCD primaire, algorithme AED, appareils AED, examen).

\section{Certificat}

L'association Swiss Resuscitation Council (SRC) crée un certificat qui est remis à chaque participant à un cours AED après réussite de l'examen écrit et pratique. Le certificat est établi par l'instructeur du cours. La participation à un cours de répétition $\mathrm{AED}$ et la recertification sont recommandées tous les deux ans.

\section{Instructeurs AED}

L'attestation du cours réussi d'instructeur AED est nécessaire. Les instructeurs médecins et non-médecins des cours AED doivent remplir plusieurs conditions.

\section{Contrôle qualité}

La direction des cours AED est confiée à un médecin compétent en matière de mesures de réanimation, habilité par le SRC à la direction médicale de ces cours. La compétence professionnelle de l'instructeur forme la condition de la reconnaissance du cours par le SRC. L'instructeur médecin est responsable de la teneur des cours.

\section{Recommandations pour le cours}

\begin{tabular}{lc}
\hline Thèmes, teneur & $\begin{array}{c}\text { Durée } \\
\text { (min.) }\end{array}$ \\
\hline $\begin{array}{l}\text { Bienvenue et communications pratiques } \\
\text { Algorithme international ACLS (ILCOR), }\end{array}$ & 5 \\
\hline $\begin{array}{l}\text { chaîne de survie (chain of survival), } \\
\text { importance de la défibrillation précoce }\end{array}$ & 10 \\
\hline ABC primaire (Basic Life Support) & 60
\end{tabular}




\begin{tabular}{lc}
$\begin{array}{l}\text { Thèmes, teneur } \\
\text { Connaissance des appareils AED, exercices } \\
\text { pratiques }\end{array}$ & $\begin{array}{l}\text { Durée } \\
\text { (min.) }\end{array}$ \\
\hline $\begin{array}{l}\text { Algorithme AED } \\
\text { Dangers et situations particulières: eau, } \\
\text { matériaux explosifs, dispositifs transder- } \\
\text { miques à la nitroglycérine, femmes } \\
\text { enceintes, stimulateurs cardiaques, défi- } \\
\text { brillateurs internes à déclenchement } \\
\text { automatique (ICD) }\end{array}$ \\
\begin{tabular}{l} 
Examens écrits et pratiques \\
\hline $\begin{array}{l}\text { Evaluation, débat } \\
\text { Temps nécessaire }\end{array}$
\end{tabular} \\
\hline
\end{tabular}

\section{Références}

1 Guidelines 2000 for Cardiopulmonary Resuscitation and Emergency Cardiovascular Care. An International Consensus on Science. Resuscitation 2000;46:1-448.

2 American Heart Association in Collaboration with the International Liaison Committee on Resuscitation. Guidelines 2000 for Cardiopulmonary Resuscitation and Emergency Cardiovascular Care. Circulation 2000;102 (Suppl I):1-384.

3 American Heart Association: Heartsaver AED Manual. Dallas, TX, 2001.

4 Directives de l'IAS/IVR (Interassociation de sauvetage) pour médecins concernant la délégation des compétences médicales à du personnel nonmédecin dans le domaine du sauvetage, IV, 1997.

5 Directives de l'IAS/IVR concernant la défibrillation dans le domaine du sauvetage par des nonmédecins, III, 1995.

\section{Buchbesprechung}

«Nichts ist geschaffen, das nit dem

Menschen zu ergründen sey». Paracelsus als Geisterkenner

Theophrast von Hohenheim. Das Buch von den Nymphen, Sylphen, Pygmäen, Salamandern und den übrigen Geistern. Faksimile der Ausgabe Basel 1590. Übertragen und mit einem Nachwort versehen von Gunhild Pörksen.

2. Auflage. Marburg an der Lahn: BasiliskenPresse; 2003. 104 S., 1 Abb., Fr. 55.-.

ISBN 3-925347-68-2

Ein kleines Buch mit grosser Wirkung: Von der hier vorzustellenden Schrift über die Elementargeister haben sich nicht nur Shakespeare, Goethe und die deutschen Romantiker, sondern auch Autoren des 20. Jahrhunderts wie Ingeborg Bachmann und Ezra Pound inspirieren lassen. Erst mit Sigmund Freud hat wohl wieder ein Arzt so viel Einfluss auf die schöne Literatur gewonnen.

Der Autor, Philippus Aureolus Theophrastus Bombastus von Hohenheim, genannt Paracelsus, lebte von 1493/94 bis 1541, also zu Beginn der Neuzeit. Als Arzt und Naturphilosoph, der unorthodoxe Ideen vertrat, führte er ein unstetes Wanderleben. Trotzdem ist eine erstaunliche Fülle von Schriften unter seinem Namen überliefert. Nicht alle dieser Werke stammen tatsächlich von ihm - doch für die
Schrift über die Elementargeister ist seine Autorschaft gesichert, auch wenn das Manuskript wie viele andere erst nach seinem Tod zum Druck kam. Der berühmte Schweizer zeigt sich hier nicht als praktischer Arzt und auch nicht als Alchemist, sondern als beschreibender und ordnender Naturinterpret.

\section{Göttliche oder teuflische Geschöpfe?}

Wer sind denn diese «Nymphen, Sylphen, Pygmäen, Salamander und übrigen Geister», die im Titel genannt werden? In unserem modernen Weltsystem haben sie keinen Platz mehr. Doch Paracelsus geht mit Akribie und systematisierendem Blick daran, diese imaginären Geschöpfe in ihrem Wesen und Treiben darzustellen. Für ihn sind sie real existent und ebenso Geschöpfe Gottes wie die Menschen. Mit letzterem stellte er sich gegen die Ansicht seiner Zeitgenossen. Diese glaubten zwar auch an solche Gestalten, sahen sie jedoch als Teufelswesen an. Bei der Beschreibung der Nymphen oder Wasserfrauen behauptet der Katholik Paracelsus sogar kühn, dass es «in der Römischen Kirche mehr Abgötterei gibt als bei all diesen Frauen oder Hexen zusammen».

Die von Paracelsus beschriebenen Geister verteilen sich auf die vier antiken Elemente: Während die Nymphen im Wasser leben, sind die Salamander im Feuer, die Pygmäen in der 
Erde und die Sylphen in der Luft beheimatet. Ihnen gehören drei Viertel der Erde, während der Mensch - auch er ein Luftwesen - nur ein Viertel bewohnt. Paracelsus relativiert damit die Einzigartigkeit und die zentrale Rolle des Menschen in der Schöpfung. Neben Adam hat Gott offenbar noch andere Wesen erschaffen, die Vernunft besitzen.

Wie man zu einer Seele kommt

Die Elementargeister sind keine «reinen» Geister, sondern aus Fleisch und Blut, also auch sterblich wie der Mensch. Sie sind uns ähnlicher als die Tiere. Sie haben, so betont der Arzt Paracelsus, dieselben Krankheiten wie wir, sie arbeiten, tragen Kleider, können sprechen. Ihnen fehlt nur ein entscheidendes Merkmal: die Seele. Paracelsus wiederholt diese Unterscheidung mehrmals, als ob er sich selbst davon überzeugen müsste. In ihrem informativen Nachwort erklärt die Paracelsus-Kennerin Gunhild Pörksen den Grund: Paracelsus kam offenbar aus philosophischen Überlegungen erst hier im Spätwerk zu diesem Schluss. Doch scheint er die Seelenlosigkeit der «wilden Leute» zu bedauern. Und so öffnet er ihnen einen Weg zur Rettung: Wegen ihrer Ähnlichkeit mit dem Menschen können sich die meisten dieser Wesen mit menschlichen Partnern verbinden und sogar Kinder zeugen. Durch eine treue eheliche Verbindung verlieren sie ihren Geisterstatus und werden zu richtigen Menschen mit einer unsterblichen Seele - ein Thema, das dann in Andersens «Kleiner Meerjungfrau» und anderen Undinenmärchen behandelt worden ist. Die Elementargeister gebären aber auch «Monstra», das sind nach Paracelsus Gestalten wie die Riesen, Zwerge und Sirenen.

\section{Lob der Schöpfung}

Das reichhaltige und mit rhetorischem Geschick geschriebene Werk will zeigen, dass Gottes Schöpfung grösser ist als gemeinhin gedacht. Zugleich vollzieht Paracelsus eine Relativierung der Bedeutung des Menschen. Mit deutlicher Sympathie nimmt er Stellung für die nicht-adamitischen Geschöpfe und erklärt sie zu Wunderwerken der Natur. Damit haben sie ihre Daseinsberechtigung neben dem Menschen und erinnern ihn daran, seine Seele als Gottesgeschenk zu pflegen.

Der sorgfältig gemachte Band enthält das Faksimile des Erstdruckes von 1590 und eine hilfreiche Übersetzung des Originaltextes ins heutige Deutsch, die ebenfalls von Gunhild Pörksen stammt. So lässt sich der von Paracelsus aus antiken und volkstümlichen Überlieferungen zusammengetragene und systematisch aufbereitete Schatz auf einfache Weise wieder geniessen.

Margrit Wyder, Zürich 